\title{
Numerical investigation of drag reduction in a Class 5 medium duty truck
}

\author{
M. Norouzi ${ }^{1}$ * M.A. Pooladi ${ }^{2}$ and M. Mahmoudi ${ }^{3}$ \\ ${ }^{1}$ Mechanical Engineering Department, Shahrood University of Technology \\ *Email: mnorouzi@shahroodut.ac.ir \\ Phone: +989123726933 \\ ${ }^{2}$ Islamic Azad University of Kish International Branch \\ ${ }^{3}$ Mechanical Engineering Department, Isfahan University of Technology
}

\begin{abstract}
In the present paper, the three-dimensional numerical investigation of Class 5 medium duty trucks (based on a production of Volvo Company) is carried out. The aim is to investigate and provide additional insights about the drag reduction methods in medium duty trucks. The flow field and pressure distribution around the truck were simulated using the Finite Volume Method. The Simple algorithm was employed to couple the pressure and momentum. A constant velocity of $30 \mathrm{~m} / \mathrm{s}$ was set in the inlet, the non-slip condition in conjugation with a wall function were used on the truck and ground surfaces, and the pressure-outlet was applied at the outlet. For the turbulent regime, the well-known standard k- $\varepsilon$ model was used to simulate the turbulent flow characteristics. The effects of vortexes around the vehicle on the drag coefficient were studied. Also, some passive devices such as standard and large windbreakers, convex roof, and the axial channel were considered for drag reduction at a high velocity $(30 \mathrm{~m} / \mathrm{s})$ and standard atmospheric conditions $\left(\mathrm{T}=25^{\circ} \mathrm{C}, \mathrm{p}=1 \mathrm{~atm}\right)$. The results showed that the large windbreakers and covering the gap between the trailer and the container are not suitable successors for standard windbreakers. Furthermore, it was found that the convex roof is a suitable passive or active device for notable drag reduction (25\%). Some recommendations for future works might include investigating the effect of combinations of different devices on the drag reduction, studying the different underbody devices like side skirts, and using more sophisticated turbulent models such as large eddy simulation.
\end{abstract}

Keywords: Aerodynamic, Drag reduction; Class 5 truck.

\section{INTRODUCTION}

In the recent years, the fuel consumption reduction became a growing demand for goods transportation by trucks [1-6]. More than anything else, the vehicle aerodynamic performance is determined by the drag force applied to the vehicle, which directly affects the truck fuel consumption [2]. It is known that the aerodynamic forces increase with truck velocity so that at high speeds it becomes the main cause of resistance against the motion [7]. Addition of devices installed on a truck is an effective way to reduce the drag coefficient. According to the wide use of trucks for shipping goods across countries, even small reduction in aerodynamic drag will lead to significant reduction in fuel consumption and thereby reduction in the truck emissions [8]. Thus, expanding our knowledge about the effects of various devices on the drag coefficient and the changes are induced on the flow field around the trucks becomes crucial in designing future optimal drag reducing 
devices. Although experimental data obtained from the wind tunnel are remarkably important, utilization of the numerical methods to simulate the flow field around the vehicles leads to decrease in time cost and number of wind tunnel experiments, extensively [9]. Despite so many advances in the numerical methods, the accurate and valid simulation of flow around the trucks is not simple regarding to the different phenomena in the turbulent flow field as well as the geometric complexities. Flow separation and vortex formation occur in the turbulent flow around the trucks $[10,11]$. Thus, the three-dimensional simulation of this kind of problems requires strong meshing tool, reliable solution algorithms, and appropriate computer resources.

Various experimental and numerical studies have been performed on the topic of drag reduction in heavy and light trucks (i.e.[12, 13]). Hyams et al. [14] have carried out numerical solutions of the unsteady Reynolds-averaged Navier-Stokes equations using a parallel flow solver to investigate the effect of unsteady aerodynamics flow on the fuel economy of class 8 trucks, they have discussed about the effects of yaw angle, spinning wheels, cab extenders, splitter plates, and base flaps on the flow field around the truck. Xiao and Yong [15] have studied the truck-induced air flow. They have used the results of their numerical investigation to assess the effectiveness of the installed hash symbol shaped fence on the truck. They found that the hash symbol fence plays its role better than those of the original model, due to its aerodynamic characteristics. Hu et al. [16] have carried out numerical simulations to study the variation of aerodynamic loads and flow fields in heavy-duty trucks while crossing a viaduct with $1.1 \mathrm{~m}$ high fences in a cross wind at the velocity of $20 \mathrm{~m} / \mathrm{s}$. They employed the SST k- $\omega$ turbulence model to simulate the flow field around the truck. Their results showed that using a fence weakens the side force. Also, they found that when the truck enters the viaduct, the direction of side force changes.

Verzicco et al. [17] have simulated the flow around an idealized road vehicle at Reynolds numbers up to $10^{5}$ using the large eddy simulation. They have analyzed the effect of the Reynolds number and the wake modifications produced by drag reduction devices. They compared their results with the available experimental data. Yang et al. [18] have numerically studied the role of external sun visor on aerodynamic drag on a heavy-duty truck. They solved the flow field around the truck model using the RANS equations in conjugate with SST k- $\omega$ turbulence model. They showed that the sun visor has a great influence on the local flow field, but relatively small effect on the global flow field. Selenbas et al. [19] have performed a computational fluid dynamics study to optimize the cabin geometry and its different parts for reducing the drag coefficient. Also, they have carried out an experimental investigation in a wind tunnel to validate the numerical results using a $1 / 5$ scale truck model. They showed that the detailed CFD analyses provide a valuable tool for the fine tuning of the cabin geometry and its accessories. Khalesi Doost and Seif Zadeh Yazdi [20] have used the FLUENT and the flow field governing equations to simulate the flow field around vehicles. They have used the results of aerodynamic analyses of the streamlines, vortexes, and pressure distribution on the vehicle structure to present a method to reduce the drag force. They reached to $23 \%$ drag reduction by channeling the air from the front bumper to rear one as well as decreasing the size of vortex behind the vehicle. Skea et al. [21] have presented various methods regarding the vehicle's aerodynamics and numerically examined them to analyze different types of geometries. Hwang et al. [22] have performed wind tunnel tests and numerical simulations to investigate the effects of side skirt on the drag reduction in heavy-duty trucks. They presented two different types of side skirts. From the results of wind tunnel, they found that the presence of side skirts leads to more than $5 \%$ drag 
reduction. Spike et al. [23] have proposed five different external flow devices to reduce the drag on a pickup truck. They numerically simulated the flow field around the truck in order to investigate the effect of each device on the drag coefficient. They found that the most efficient external drag reducing device was side panels.

In the present paper, the effects of vortexes around the vehicle and addition of some passive devices such as standard and large windbreakers, convex roof, and the axial channel on the drag coefficient for a class 5 medium duty truck were studied. The main objectives of this study are to identify the contributions of large windbreaker, covering the gap between the tractor and container, axial channel, and convex roof in drag reduction in medium-duty trucks.

\section{METHODS AND MATERIALS}

\section{Governing Equations}

The steady state turbulent flow around a class 5 truck of VOLVO Company named FH480 is studied. The flow field was modeled using the FLUENT 6.3.2 as the CFD solver. The governing equations for conservation of mass and momentum of air have been solved in the Cartesian coordinates. The mass conservation (continuity) is:

$$
\frac{\partial\left(\rho u_{i}\right)}{\partial x_{i}}=0
$$

and the momentum equation can be written as [24]:

$$
\frac{\partial}{\partial x_{j}}\left(u_{i} u_{j}\right)=-\frac{1}{\rho} \frac{\partial p}{\partial x_{i}}+\frac{\partial}{\partial x_{j}}\left[v\left(\frac{\partial u_{i}}{\partial x_{j}}+\frac{\partial u_{j}}{\partial x_{i}}\right)\right]+\frac{\partial}{\partial x_{j}}\left(-\overline{u_{i}^{\prime} u_{j}^{\prime}}\right)
$$

where $\rho$ and $\mu$ are the air density and dynamic viscosity at atmospheric conditions, respectively $\left(\rho=1.225 \mathrm{~kg} \cdot \mathrm{m}^{-3}, \mu=1.789 \times 10^{-5} \mathrm{~kg} \cdot \mathrm{m}^{-1} \cdot \mathrm{s}^{-1}\right.$ ). The Reynolds stress term in the momentum equation, i.e. $-u_{i}^{\prime} u_{j}^{\prime}$, is modeled using by the standard $k-\varepsilon$ model in conjugate with the Boussinesq hypothesis [24]:

$$
-u_{i}^{\prime} u_{j}^{\prime}=v_{t}\left(\frac{\partial u_{i}}{\partial x_{j}}+\frac{\partial u_{j}}{\partial x_{i}}\right)
$$

where $v_{t}$ is the eddy viscosity and can be achieved by,

$$
v_{t}=C_{\mu} k^{2} / \varepsilon
$$

The kinematic energy of turbulence and the dissipation rate of the turbulence kinetic energy can be written as [24]:

$$
u_{j} \frac{\partial k}{\partial x_{j}}=\tau_{i j} \frac{\partial u_{i}}{\partial x_{j}}-\varepsilon+\frac{\partial}{\partial x_{j}}\left[\left(v+\frac{v_{t}}{\sigma_{k}}\right) \frac{\partial k}{\partial x_{j}}\right]
$$




$$
u_{j} \frac{\partial \varepsilon}{\partial x_{j}}=C_{\varepsilon 1} \frac{\varepsilon}{k} \tau_{i j} \frac{\partial u_{i}}{\partial x_{j}}-C_{\varepsilon 2} \frac{\varepsilon^{2}}{k}+\frac{\partial}{\partial x_{j}}\left[\left(v+\frac{v_{t}}{\sigma_{\varepsilon}}\right) \frac{\partial \varepsilon}{\partial x_{j}}\right]
$$

where the closure coefficients read as follows:

$$
C_{\varepsilon 1}=1.44, \quad C_{\varepsilon 2}=1.92, \quad \sigma_{\varepsilon}=1.3, \quad \sigma_{k}=1.0, \quad \operatorname{Pr}_{t}=0.9
$$

The drag coefficient can be calculated as follows:

$$
C_{D}=\frac{F_{\text {total }}}{\rho V / 2}
$$

where $F_{\text {total }}=F_{\text {pressure }}+F_{\text {viscous }}$ and $\mathrm{V}$ is the far field air velocity.

\section{Numerical Method, Boundary and Initial Conditions}

The FLUENT software uses Finite Volume Method to solve Eqs (1)-(7). Simple algorithm was employed to couple the pressure and momentum, and the second-order upwind scheme was utilized for spatial discretization in all equations. The relaxation factors for $\mathrm{P}, \mathrm{U}, \mathrm{k}$, and $\varepsilon$ were $0.3,0.7,0.7$, and 0.7 , respectively. The convergence criterion was defined as the maximum relative error between of variables in two successive iterations to be less than $10^{-7}$.

In the case of boundary conditions, a constant velocity of $30 \mathrm{~m} / \mathrm{s}$ has been set at the inlet, the turbulent intensity, turbulence kinetic energy and the turbulence dissipation rate at the inlet are given by [24],

$$
I=\frac{\sqrt{\overline{u^{\prime 2}}}}{U_{e}}=0.16\left(R e_{D}\right)^{-1 / 8} \quad k=\frac{3}{2}\left(U_{e} I\right)^{2} \quad \varepsilon=C_{\mu}^{3 / 4} \frac{k^{3 / 2}}{l}
$$

where $C_{\mu}=0.09$ and the length scale is defined as $l=0.07 L$. For the ground the solid surfaces the non-slip condition in conjugate with a wall function has been applied [25]. At the outlet face, the pressure-outlet condition has been applied.

\section{Grid Generation and Grid-Independent Study}

The geometry of the class 5 medium duty truck (FH-480) have been provided and imported to Gambit for mesh generation. The overall dimensions are $150 \mathrm{~m} * 24 \mathrm{~m} * 16 \mathrm{~m}$. The truck length (L) is around $16.6 \mathrm{~m}$. The domain is divided to four calculation zones for near truck region, middle region, and far field regions (Figure 1). The mesh was generated using hexagonal grids with a growth rate of 1.2 for the near truck region. The minimum length of grids near the wall is about $0.05 \mathrm{~mm}$, while the grid size near the far field boundaries reaches to the maximum value of $5 \mathrm{~mm}$. The grids around the vehicle and on a slice in the middle of the domain are shown in Figure 2.

In order to reach a solution which is independent of the grid size, different sizes of the numerical grids were investigated. Table 1 shows the value of the drag coefficient for different grid numbers. As it can be seen in Table 1, the variation of drag coefficient versus the grid numbers for the grid numbers larger than 5 million grids becomes less than $0.1 \%$. For insurance, 7 to 8 million of grids were used for different scenarios in our 
CFD simulations. The solver is validated via solving a similar problem for a class 8 truck of Volvo Company, the drag coefficient was estimated around 0.5119 for our simulation and reported 0.50 by Hakansson [1].

Table 1. Drag coefficient in terms of grid numbers for FH-480 without windbreaker.

\begin{tabular}{cc}
\hline Grid Numbers & Drag Coefficient \\
\hline 1245023 & 0.6822 \\
3101715 & 0.7014 \\
5223854 & 0.7193 \\
7334230 & 0.7205 \\
9215117 & 0.7209 \\
11013781 & 0.7213 \\
\hline
\end{tabular}

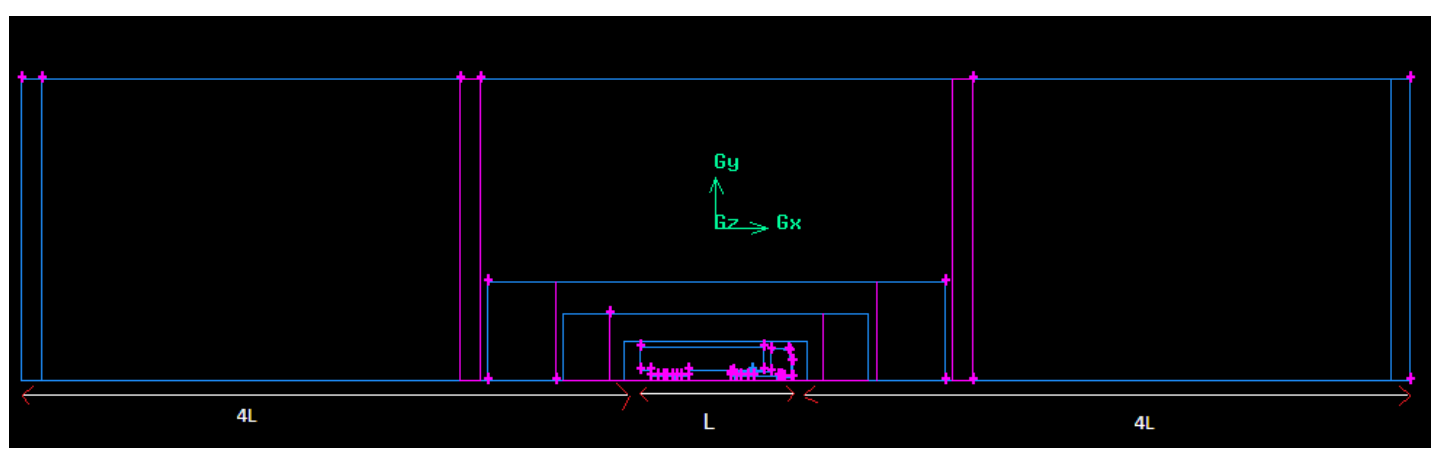

Figure 1. The domain divided into four calculation zones for near truck region, middle region, and far field regions.

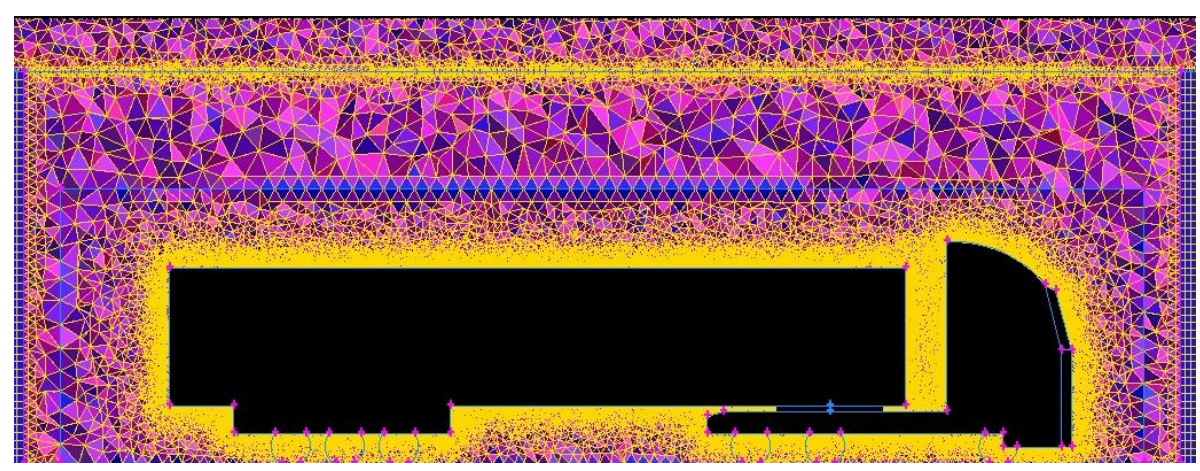

Figure 2. Grid around the truck.

\section{RESULTS AND DISCUSSION}

The following sections are devoted to the investigation of the effects of large and standard windbreakers, covering the gap between the trailer and container, axial channel, and the convex roof on the flow field and the drag reduction in a class 5 medium duty truck. Figure 3 shows the static pressure distribution and the vector plot of velocity field around a class 5 truck without any expanders. In this case, the projected area is $7.7 \mathrm{~m}^{2}$. As expected, the static pressure is large in nose region and there are mainly three wake regions located at the behind of tractor, under the trailer and behind the trailer (i.e. Region 1, Region 2, and Region 3, respectively). The vortexes in these three regions are created 
due to the flow separation. The velocity of the air and the shape of truck affect the size of these vortexes [11]. A low-pressure is created behind the trailer when the separation occurs and the air is sucked into the Region 3 from all the sides. Also, the underbody lowpressure region (Region 2) is created when the air flowing under the tractor is separated at the tractor's rear. As it can be seen in Figure 3, a low- pressure large vortex is formed between the tractor and the container. The reasons and the ways we can decrease the undesirable effects of the presence of these vortexes are discussed in following sections. The static pressure difference between front and behind of the truck is about $0.552 \mathrm{kPa}$. The size of the wake region located at behind of trailer is the largest and it has the main contribution in drag force and as a result, it plays a key role in fuel consumption [12]. The skin drag coefficient and pressure drag coefficient are 0.08 and 0.64 , respectively. The drag coefficient is 0.72 which is a large value for a truck.

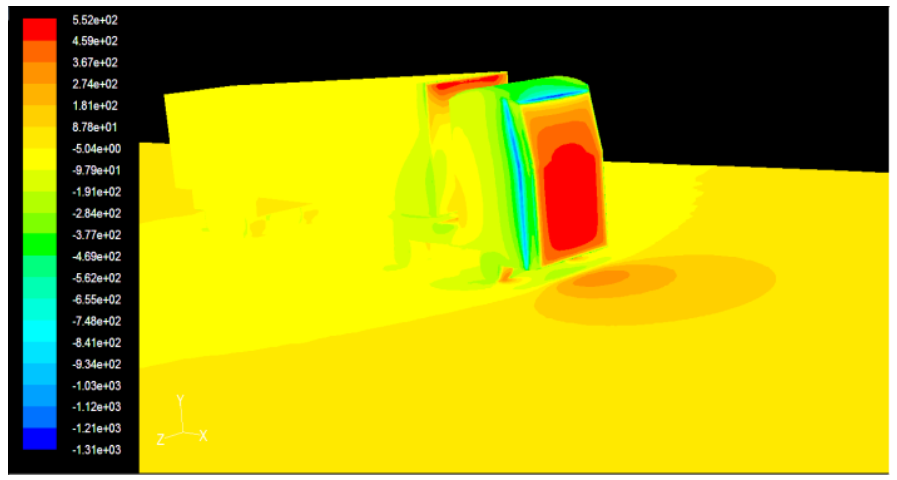

(a)

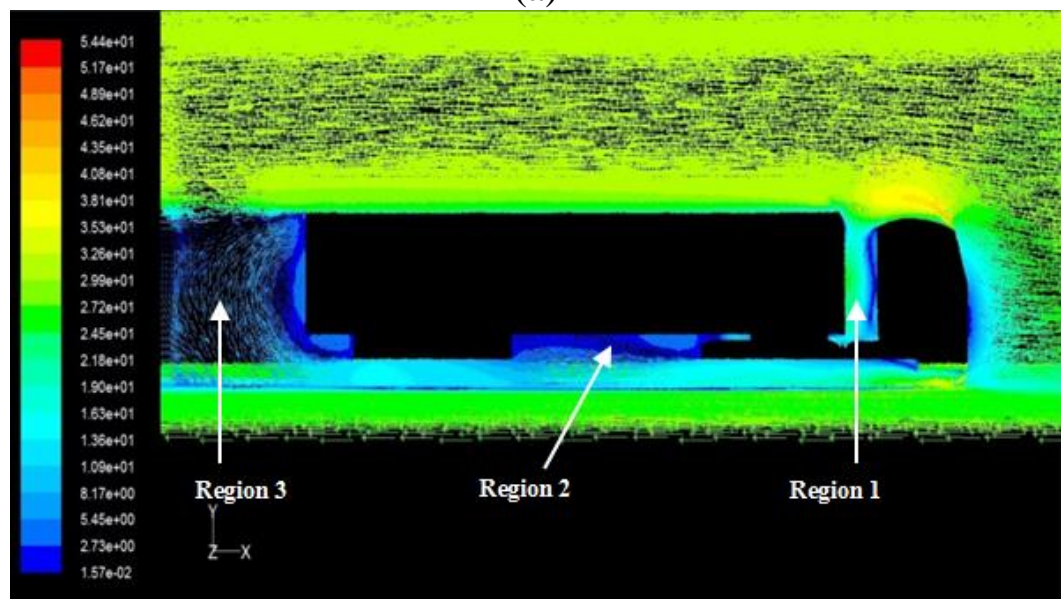

(b)

Figure 3. (a) The static pressure distribution and (b) vector plot of velocity field around the truck without any extenders.

\section{Truck with Standard Windbreaker}

In order to eliminate the created vortexes between the trailer and container, a standard windbreaker is added on the top of the trailer. The standard windbreaker was designed and produced by Volvo Company and it is used in the FH-480 model. Figure 4 shows the static pressure distribution and the velocity field around the vehicle. As it is shown in Figure 4(b), the step between the trailer and the container is removed and the vortices located in the gap become weaker than the case without windbreaker, considerably. First, the air stream hits the front of the truck and creates a relatively large high-pressure region 
there. Some of the air passes over the cab and the rest flows through the underbody space [26]. The low-pressure region in the tractor-trailer gap is caused mainly due to the wake that is formed when the flow separates at the trailing edge of the cab's roof. By comparing the pressure distributions and velocity fields in Figures 3 and 4, it can be seen that the windbreaker directs the flow over the tractor-trailer gap and decreases the flow unsteadiness and postpones the separation at the leading edge of the cab [27, 28]. The maximum static pressure on the truck surface is $0.574 \mathrm{kPa}$ which is located in front of the trailer. The viscous drag coefficient and the pressure drag coefficient for this case are 0.08 and 0.56 , respectively. In comparison with a truck without windbreaker, using the standard windbreaker results in a reduction in drag coefficient by $11 \%$ to 0.64 .

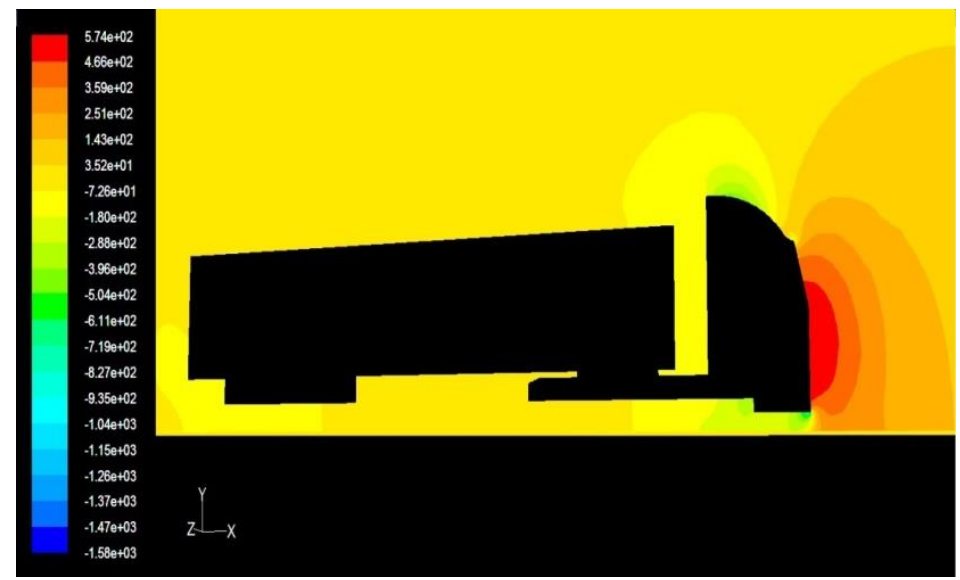

(a)

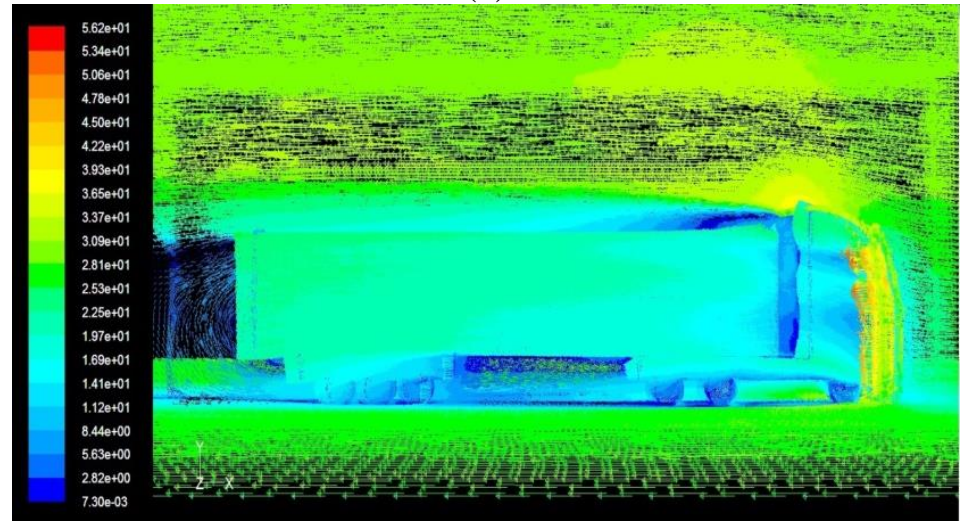

(b)

Figure 4. (a )The static pressure distribution and (b) the velocity field around the vehicle with standard windbreaker.

\section{Truck with Covered Gap}

The second method of drag reduction is to cover the gap between the trailer and container with surfaces. As it was shown in Figure 3, there are some vortices in this gap, but using a cover over this gap results in vanishing this low-pressure region. Figure 5 shows the vector plot of velocity field for a truck with covered gap. It can be seen that the vortices between the tractor and container are eliminated. As it was noted, the low-pressure region between the tractor and trailer is formed mainly because of flow separation at the trailing edge of the cab. Covering the tractor-trailer gap allows the flow to remain attached to the truck surface and provide the possibility of a smooth transition of air over the gap. Thus, one of the main causes of resistance against the truck motion can be attenuated or even 
eliminated [27]. In this case, the maximum static pressure is $0.576 \mathrm{kPa}$. Here, the skin friction is slightly increased $\left(C_{D, \text { Friction }}=0.09\right)$ and the pressure coefficient is reduced to 0.57. This increase in skin friction is due to the addition of extra surfaces to the truck which cover the tractor-trailer gap. Eliminating or attenuating the low-pressure region inside the gap causes an $8 \%$ drop in drag coefficient to 0.66 . Although, in this case, the addition of extra surfaces increased the friction resistance against the truck motion, the overall resistance is lower than the case without any drag-reducing devices.

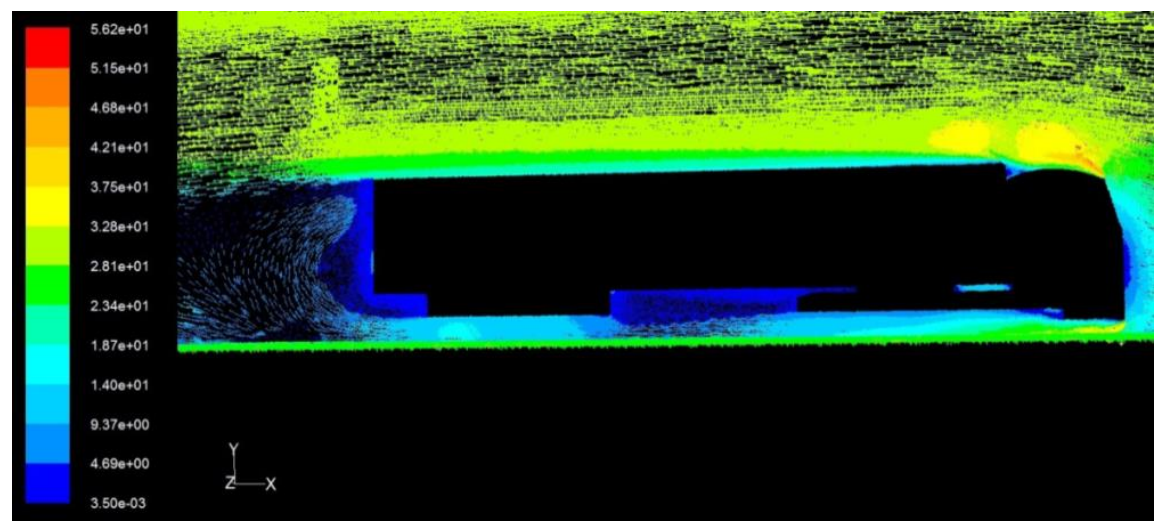

Figure 5. The vector plot of velocity field for the truck with covered gap.

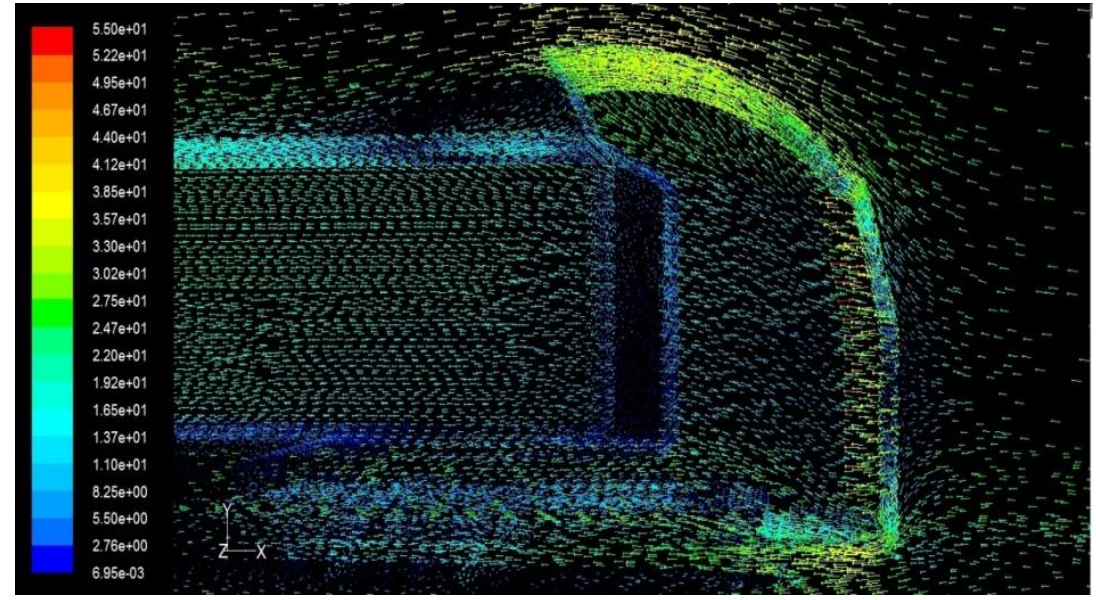

Figure 6 . The vector plot of velocity field near the truck with large windbreaker.

\section{Truck with Large Windbreaker}

As the third method of drag reduction, a large windbreaker was considered which completely covered the gap between the tractor and container. Figure 6 shows the vector plot of velocity field near the truck for this case. As it can be seen in Figure 6, although the vortices in the gap are eliminated, there is a relatively large vortex on the container which provides a low-pressure region. The reason is that as the flow approaches the rear edge of the large wind breaker, it separates there from the truck surface and forms a lowpressure region above the container and behind the wind breaker. In fact, the rear edge of wind breaker acts like a backward step in a channel which leads to flow separation at the rear edge of the backward step [29]. The skin drag on the vehicle was approximately the same as the truck without any extenders, i.e. $C_{D, \text { Friction }}=0.08$. The pressure coefficient 
was obtained to be $C_{D, \text { Pressure }}=0.55$. Thus, the drag coefficient is reduced by $12 \%$ to 0.63 when using a large windbreaker. It is evident that the large windbreaker leads to a drag reduction which is slightly smaller than a standard one. Therefore, using this kind of windbreaker has not economic justification in comparison with the standard windbreaker. This result indicates that the step between the tractor and trailer has a more important role in overall drag force and its effect can be attenuated by a standard windbreaker.

\section{Truck with Convex Roof}

A parabolic convex roof with a height of $35 \mathrm{~cm}$ is considered here. The convex roof could be useful as a passive device of drag reduction. This kind of roof is usually used in sports cars and SUVs. If the flow remains attached to a convex roof, it is possible to reduce the pressure drag force by decreasing the size of wake region behind the container. In other words, the convex roof allows the air to be attached to the surface, and at the trailing edge of the container, to flow through the low-pressure region behind the trailer and decrease the size of wake zone [30].

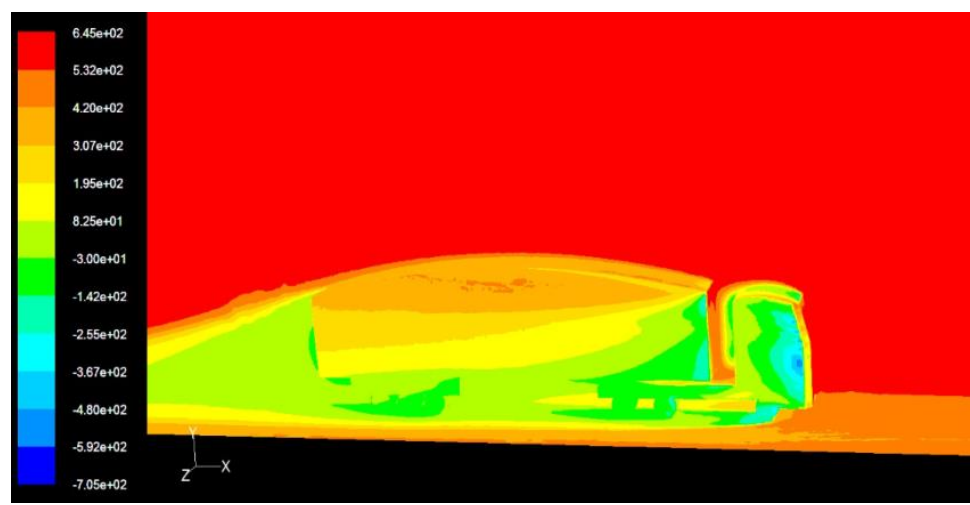

(a)

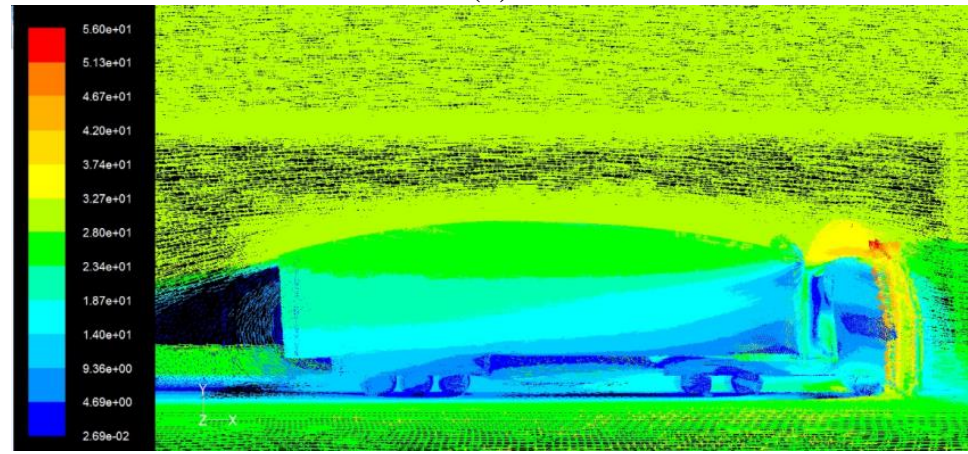

(b)

Figure 7. (a )The static pressure distribution and (b) the velocity field around the vehicle with convex roof.

Figure 7(a) presents the total pressure distribution around the class 5 medium duty truck. The maximum pressure around the vehicle is $0.645 \mathrm{kPa}$. The skin drag coefficient and pressure drag coefficient are obtained as 0.11 and 0.43 , respectively. The pressure and total drag coefficient are reduced by $33 \%$ and $25 \%$, respectively, when using a convex roof for the container. Figure 7(b) demonstrates the velocity vectors around the vehicle. According to this figure, the wake size behind the truck is reduced, considerably. Since the flow is attached to the surface, more air is transported into the wake region behind the 
trailer which increases the disturbances of the flow and size of the low-pressure region behind the truck [30]. The existence of a smaller low-pressure zone in comparison with the case without any extenders leads to decrease in pressure drag coefficient. The results indicate that using the convex roof might be a useful method for reducing the drag on a vehicle.

Figure 8 shows the effect of the height of convex roof on drag reduction. The flow is completely attached to the roof in this range of height, so the separation point is located at trailing edge of roof. The figure shows that for the attached flow, the drag is decreased by increasing the height of roof which is related to the drop in pressure drag and a decrease in the size of wake zone behind the truck. When flow is attached to the trailer's roof, increasing the convex height leads to directing more air into the low-pressure region behind the truck. In other words, the convex with a larger height directs the air into the wake region with a larger angle than the smaller one. Thus, the disturbances in this region are increased and the vortex region becomes smaller.

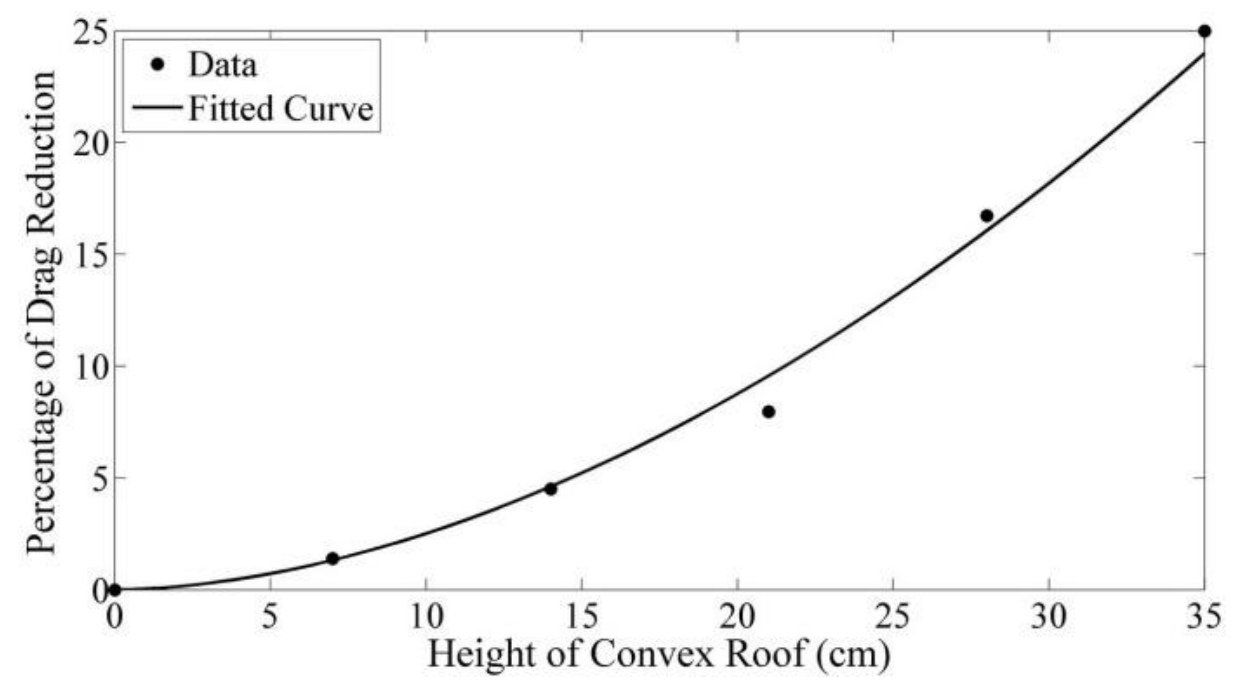

Figure 8. Percentage of drag reduction versus height of convex roof of container at $\mathrm{V}=30 \mathrm{~m} / \mathrm{s}$ for standard air condition.

\section{Truck with an Axial Air Channel}

The effect of the presence of an axial air channel which transfers the air from the truck nose, under the radiator towards the container tail is investigated. This channel is responsible for transferring the air from the high-pressure zone in front of the truck to the low-pressure region behind the container. The axial channel is split in the middle of the truck in order to distribute the air behind the container in a way that the vortices become smaller. Using the axial air channel leads to decrease in the drag force by both decreasing the pressure in front of the trailer and reducing the size of vortices behind the truck. Transporting air into the low-pressure region behind the trailer results in disturbing the wake region and making the vortexes smaller [31]. Thus, the smaller low-pressure region leads to a lower resistance against the truck motion. This method is not suitable unless the essential changes in the design of trailer are considered by automakers.

\section{Truck with Standard Windbreaker and Convex Roof}

The combined effects of the convex roof and standard windbreaker are also studied. The total pressure distribution is shown in Figure 9(a). The skin drag coefficient for this case 
is 0.12 . The pressure drag coefficient on the truck is 0.35 , and the drag coefficient is calculated as 0.47 . Thus, using a standard windbreaker along with a convex roof on a truck results in a $13 \%$ decrease in drag coefficient in comparison with the case with the only convex roof. The maximum pressure around the truck is $0.633 \mathrm{kPa}$. According to the velocity field (Figure 9b), the gap wake region is weakened and the size and intensity of the wake region behind the vehicle are decreased considerably [1]. By comparing Figures 7 and 9, it can be seen that the presence of the standard windbreaker leads to attenuating the wake region in the tractor-trailer gap by smoothly directing the flow over it. In this case, the drag coefficient is dropped about $35 \%$ relative to the truck without standard windbreaker. As an important result, the drag coefficient is reduced by $26 \%$ relative to the case with the standard windbreaker.

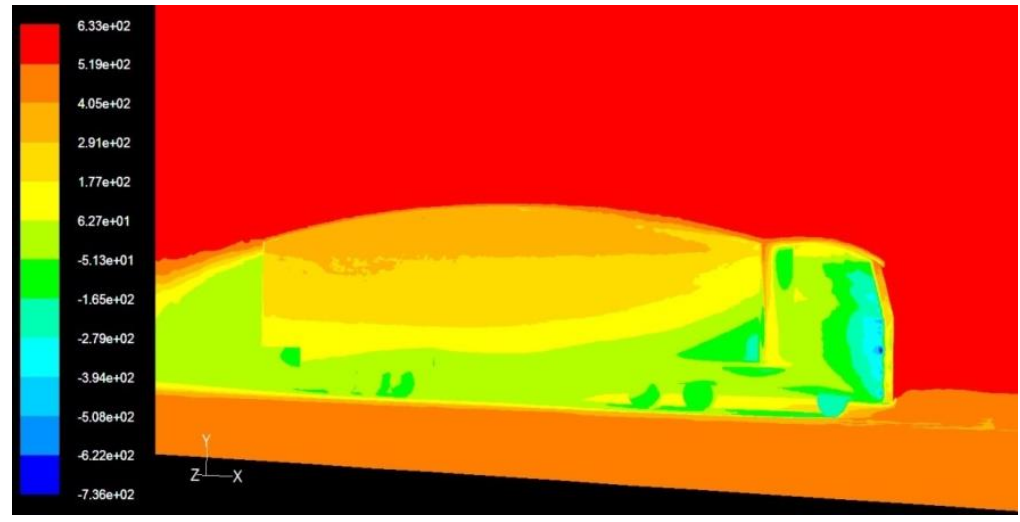

(a)

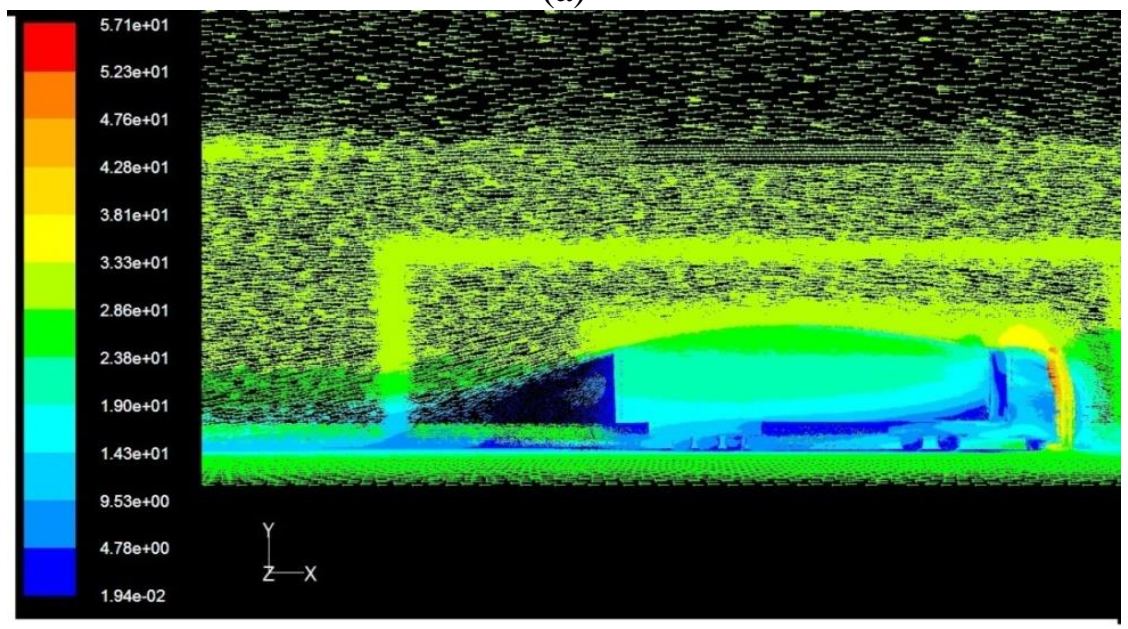

(b)

Figure 9. (a )The static pressure distribution and (b) the velocity field around the vehicle with standard windbreaker and convex roof.

\section{CONCLUSIONS}

In the present paper, the drag reduction methods in medium duty trucks were investigated. The numerical simulations were performed using FLUENT. The effect of addition of standard and large windbreakers, covering the gap between the trailer and container, axial air channel, and the convex roof to the truck FH-480 of Volvo Company were studied. The main findings of current research are summarized as follows: 
a) Large windbreak and covering the gap between the tractor and container are not suitable devices for standard windbreaker.

b) The axial channel has only 1-3\% of drag reduction in medium duty trucks and this method is not suitable unless the essential change in the design of the trailer is considered by automakers.

c) Using the convex roof is a suitable passive or active device for notable drag reduction, but traffic restrictions are the main problem against applying this method. Fortunately, this problem is less important for light and medium duty trucks.

\section{ACKNOWLEDGEMENTS}

The authors gratefully acknowledge Shahrood University of Technology and Islamic Azad University of Kish International Branch for their supports. Also, we thank the referees and the editor for useful suggestions.

\section{REFERENCES}

[1] Håkansson C, Lenngren M. CFD Analysis of Aerodynamic Trailer Devices for Drag Redution of Heavy Duty Trucks: Chalmers University of Technology; 2010.

[2] Salari K. DOE's effort to reduce truck aerodynamic drag through joint experiments and computations. LLNLPRES-401649 Presentation, Lawrence Livermore National Laboratory. 2009;28.

[3] Isaev S, Gortyshov YF, Gureev V, Opara YS, Popov I. A Method of Decreasing the Drag of a Heavy-Duty Truck with the Use of Front and Stern Board Generators of Large-Scale Vortices. Journal of Engineering Physics and Thermophysics. 2015;88:200-6.

[4] McAuliffe BR. Improving the aerodynamic efficiency of heavy duty vehicles: wind tunnel test results of trailer-based drag-reduction technologies. Report (Aerospace (Canada). Aerodynamics Laboratory), 2015-07-22; 2015.

[5] Vegendla P, Saha R, Sofu T, Hwang L-K. Comparison of aerodynamic drag and underhood thermal analysis of two heavy-duty vehicles. International Journal of Aerodynamics. 2016;5:105-24.

[6] Abas M, Said MM, Abidin SZ, Zahari I. Simulation of Fuel Economy for Malaysian Urban Driving. International Journal of Automotive and Mechanical Engineering. 2015;11:2306-16.

[7] Wang Y, Wu C, Tan G, Deng Y. Reduction in the aerodynamic drag around a generic vehicle by using a non-smooth surface. Proceedings of the Institution of Mechanical Engineers, Part D: Journal of Automobile Engineering. 2017;231:130-44.

[8] Al-Garni AM. Measurements of the cross-flow velocity field in the wake of an idealized pickup truck model using particle image velocimetry. 14th International Symposium on Applications of Laser Techniques to Fluid Mechanics Lisbon, Portugal2008. p. 1-10.

[9] Wang D, Wang Y, Han Y, Dang Y, Fan D, Li L. Numerical Simulation of the Influence of Additional Aerodynamic Devices on the Aerodynamic Drag of VanBody Truck. Proceedings of SAE-China Congress 2014: Selected Papers: Springer. 2015;15-26. 
[10] McArthur D, Burton D, Thompson M, Sheridan J. On the near wake of a simplified heavy vehicle. Journal of Fluids and Structures. 2016;66:293-314.

[11] Manay E, Ozceyhan V, Sahin B, Gunes S. Edge length effect of bluff bodies on flow structure. International Journal of Automotive and Mechanical Engineering. 2014;9:1793-1802.

[12] Mohamed-Kassim Z, Filippone A. Fuel savings on a heavy vehicle via aerodynamic drag reduction. Transportation Research Part D: Transport and Environment. 2010;15:275-84.

[13] Ortega J, Salari K. An experimental study of drag reduction devices for a trailer underbody and base. 34th AIAA Fluid Dynamics Conference and Exhibit. Portland, OR, United States.2004;1-15.

[14] Hyams DG, Sreenivas K, Pankajakshan R, Nichols DS, Briley WR, Whitfield DL. Computational simulation of model and full scale Class 8 trucks with drag reduction devices. Computers \& Fluids. 2011;41:27-40.

[15] QI X-n, LIU Y-q, DU G-s. Experimental and numerical studies of aerodynamic performance of trucks. Journal of Hydrodynamics, Ser B. 2011;23:752-8.

[16] HU X-j, Peng Q, Lei L, Peng G, WANG J-y, Bo Y. Numerical simulation of the aerodynamic characteristics of heavy-duty trucks through viaduct in crosswind. Journal of Hydrodynamics, Ser B. 2014;26:394-9.

[17] Verzicco R, Fatica M, Iaccarino G, Moin P, Khalighi B. Large eddy simulation of a road vehicle with drag-reduction devices. AIAA Journal. 2002;40:2447-55.

[18] Yang HB, Hu XJ, Li TF. Numerical analyses to investigate the impact of external sun visor on Aerodynamic drag of heavy-duty commercial truck. Applied Mechanics and Materials: Trans Tech Publ; 2014. p. 787-90.

[19] Selenbas B, Gunes H, Gocmen K, Bahceci U, Bayram B. An aerodynamic design and optimization of a heavy truck for drag reduction. ASME 2010 10th Biennial Conference on Engineering Systems Design and Analysis. Istanbul, Turkey: American Society of Mechanical Engineers; 2010. p. 121-9.

[20] Doost AK, Yazdi AMSZ. Green nature and reducing of air pollution with vehicle drag coefficient correction. Advances in Energy Engineering. 2013;1:28-33.

[21] Skea A, Bullen P, Qiao J. Underbody aerodynamics: Using CFD to simulate the airflow around a rotating wheel of a passenger car. Auto tech, Birmingham. 1999.

[22] Hwang BG, Lee S, Lee EJ, Kim JJ, Kim M, You D, et al. Reduction of drag in heavy vehicles with two different types of advanced side skirts. Journal of Wind Engineering and Industrial Aerodynamics. 2016;155:36-46.

[23] Spike CG, Finn TJ, Dubreuil EM, Wessner AK, Lee S-J. Reduction of Aerodynamic Drag on a Commercial Pickup Truck via External Flow Devices. 54th AIAA Aerospace Sciences Meeting. San Diego, California, USA2016. p. 1107.

[24] Wilcox DC. Turbulence modeling for CFD. Third ed. San Diego, California, USA: Birmingham Press; 2006.

[25] Pinzon C, Agarwal R. An Experimental and Computational Study of a Zero-NetMass-Flux (ZNMF) Actuator. 46th AIAA Aerospace Sciences Meeting and Exhibit. Reno, Nevada2008. p. 559.

[26] Wang XY, Hu XJ, Liao L, Li TF. Numerical Simulation to investigate Influence of Additional Devices on Aerodynamic Drag for Heavy-duty Commercial Truck. Applied Mechanics and Materials. 2012;209:2089-93. 
[27] Guo P, Hu XJ, Zhu YY, Fu Q, Wang XY, Fan SJ, et al. Investigation on Aerodynamic Drag Reduction of Commercial Truck Based on External Styling of Cab. Applied Mechanics and Materials. 2013;307:186-91.

[28] Das P, Tsubokura M, Matsuuki T, Oshima N, Kitoh K. Large eddy simulation of the flow-field around a full-scale heavy-duty truck. Procedia Engineering. 2013;56:521-30.

[29] Nguyen TD, Souad H. PIV measurements in a turbulent wall jet over a backwardfacing step in a three-dimensional, non-confined channel. Flow Measurement and Instrumentation. 2015;42:26-39.

[30] Antony A. Tear Drop Design of Double Decker Bus For Improved Aerodynamics: Coventry University; 2012.

[31] Yajima Y, Sano O. A note on the drag reduction of a circular cylinder due to double rows of holes. Fluid Dynamics Research. 1996;18:237-43. 